\title{
Extraits antigéniques spécifiques obtenus à partir d'Entamoeba bistolytica
}

\author{
II. Séparation et analyse de fractions spécifiques \\ à partir de l'antigène brut \\ par J. BENEX \\ (Collaboration technique Catherine Rouge)
}

Institut Pasteur, Service de Parasitologie, 25, rue du Docteur-Roux, F. 75 -Paris-15

\begin{abstract}
Résumé
Le fractionnement sur gel de Sephadex d'un antigène préparé à partir d'E. histolytica maintenues en culture bactériologiquement définie, permet la séparation de trois fractions spécifiques immunologiquement actives et comparables en réaction de diagnostic à celles obtenues à partir de souche de la même espèce maintenue en culture pure.

Ce fractionnement permet, en outre, de séparer deux fractions correspondant à la flore associée.

La sensibilité des fractions correspondant à $E$. histolytica est cependant moindre que celle de l'antigène brut mais l'emploi de celui-ci nécessite l'absorption des sérums à tester par un antigène flore associée.
\end{abstract}

\section{Summary}

Fractionation with Sephadex G 200 chromatographic column of an amoeba antigen extract from Entamoeba histolytica cultivated with associated bacteria yielded five fractions.

Three of them are specific of ameoba and react in serologic tests in the same way as these obtained from axenic cultures. The last two are in connection with associated bacteria.

Dans un précédent travail (1), nous avons rapporté l'obtention d'un antigène total à partir d'Entamoeba histolytica maintenues en culture mixte. Aujourd'hui, nous présentons les résultats du fractionnement de cet antigène complet sur colonne de gel de dextrane, dans le but d'en isoler des extraits purifiés spécifiques.

La valeur immunologique de chaque fraction est déterminée dans les réactions sérologiques déjà utilisées dans le travail précédent. 


\section{Matériel et méthode}

Nous avons choisi comme technique de fractionnement, la séparation par passage sur une colonne de Sephadex G. 200 (Pharmacia Upsala).

Les dimensions de la colonne utilisée étaient de $45 \times 2,5 \mathrm{~cm}$ avec un volume total de $225 \mathrm{ml}$.

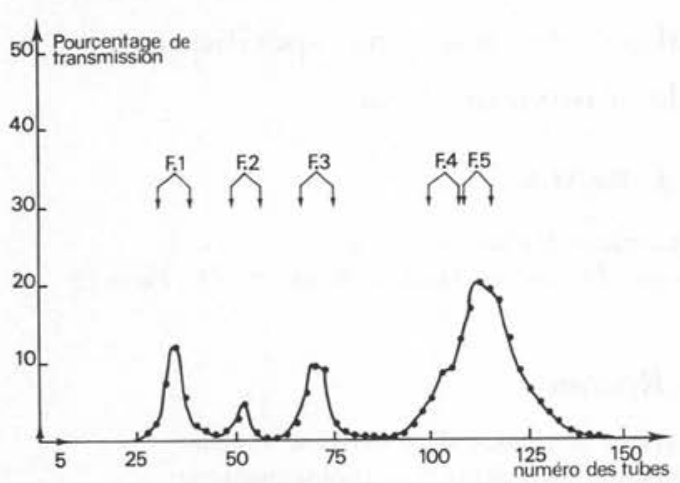

$10 \mathrm{ml}$ de l'antigène amibien brut total correspondant à environ $10 \mathrm{mg}$ de protéines sont passés sur la colonne et élués par du tampon phosphate $0,15 \mathrm{M} \mathrm{pH} \mathrm{7,2.} \mathrm{Les} \mathrm{fractions} \mathrm{sont}$ recueillies à raison de 50 gouttes par tube et la courbe est établie par une détermination au photomètre d'absorption à $280 \mathrm{mu}$.

Dans ces conditions, on obtient une courbe présentant 5 pics (courbe $\left.n^{\circ} 1\right)$.

Les contenus des tubes correspondants à la surface délimitée par ces pics sont rassemblés et on constitue ainsi cinq fractions qui sont étudiées du point de vue immunologique par les mêmes méthodes que l'antigène brut total.

Les cinq fractions sont concentrées sur carbovax, afin de les ramener à une même teneur en protéines $(0,45$ à $5 \mathrm{mg} / \mathrm{ml}$ selon les réactions sérologiques utilisées).

\section{Contrôle de l'activité immunologique des fractions}

\section{I) Test au latex.}

Première DÉTERmination QUALITATIVE

\begin{tabular}{|c|c|c|c|c|}
\hline Ag. Sérum & $\begin{array}{l}\text { Anti-flore } \\
+ \text { amibe }\end{array}$ & Anti-flore & $\begin{array}{l}\text { Anti-amibes } \\
\text { saturé flore }\end{array}$ & $\begin{array}{l}\text { Sérum } \\
\text { négatif }\end{array}$ \\
\hline F I $\ldots \ldots \ldots$. & $\begin{array}{l}\text { belle aggl. } \\
++++\end{array}$ & aggl. ++ & aggl. ++++ & - \\
\hline F II $\ldots \ldots \ldots \ldots$ & aggl. ++ & ++ & - & - \\
\hline F III $\ldots \ldots \ldots$ & aggl. +++ & ++ & - & - \\
\hline F IV $\ldots \ldots \ldots$ & aggl. $\underset{(+)}{++}+$ & 一 & + & - \\
\hline F V ..... & aggl. ++ & aggl. $\mp$ & - & - \\
\hline
\end{tabular}

Une détermination quantitative en comparaison avec l'antigène brut total est alors effectuée. 

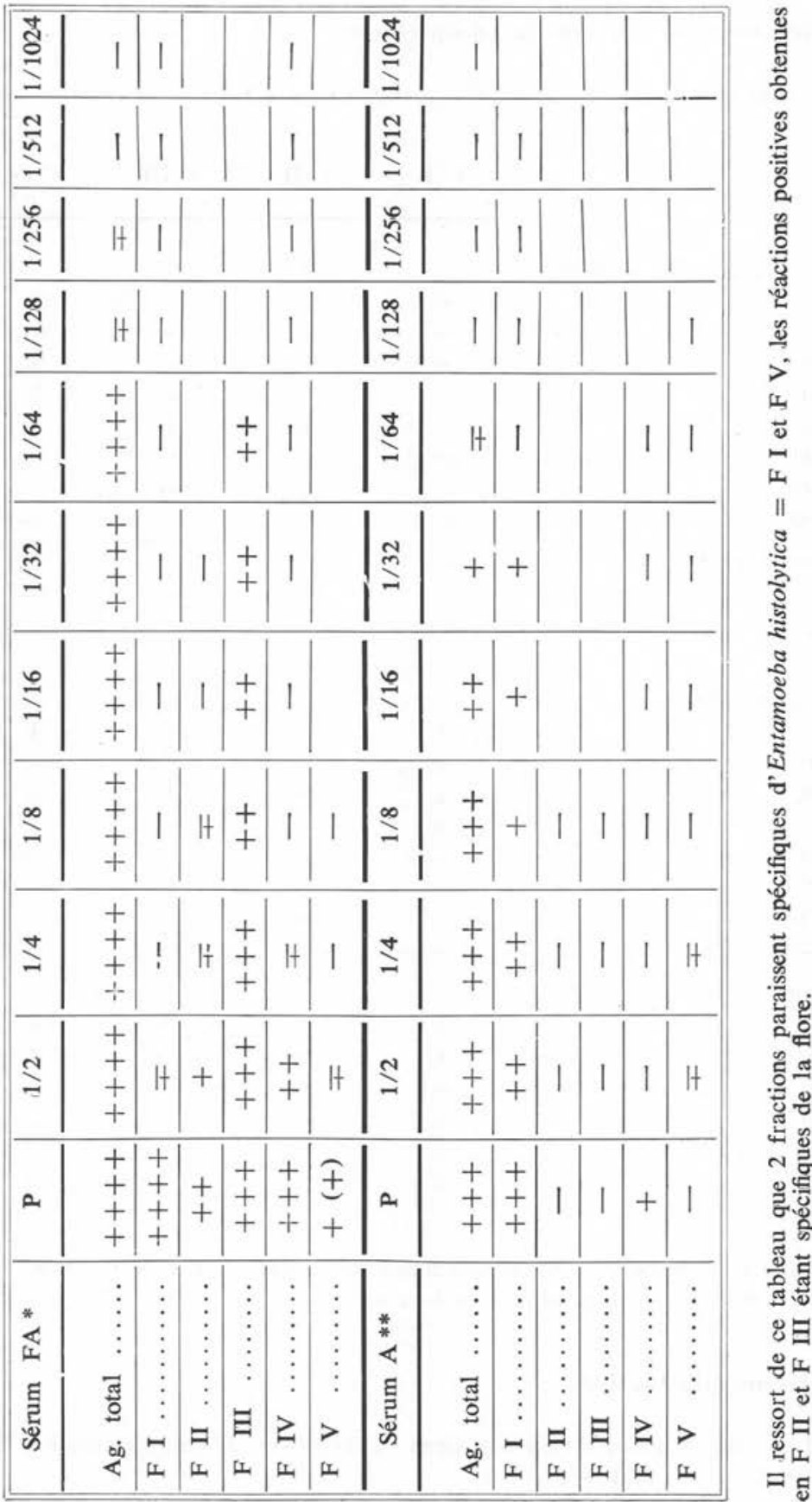
II) Réaction de fixation du complément.

Après titrage du complément, on obtient les résultats suivants :

\begin{tabular}{|c|c|c|c|c|c|}
\hline & F I & F II & F III & F IV & F V \\
\hline \multicolumn{6}{|l|}{ Sérum anti-amibes + flore : } \\
\hline $1 / 5 \quad \ldots \ldots \ldots \ldots \ldots \ldots \ldots$ & + & + & + & + & + \\
\hline $1 / 10 \quad \ldots \ldots \ldots \ldots \ldots \ldots$ & + & + & + & + & + \\
\hline $1 / 20 \ldots \ldots \ldots \ldots \ldots \ldots \ldots$ & + & + & + & + & + \\
\hline $1 / 40 \ldots \ldots \ldots \ldots \ldots \ldots$ & + & + & + & + & + \\
\hline $1 / 80 \ldots \ldots \ldots \ldots \ldots \ldots$ & + & + & + & + & + \\
\hline $1 / 160 \quad \ldots \ldots \ldots \ldots \ldots \ldots$ & + & $\mp$ & $\mp$ & - & - \\
\hline $1 / 320 \quad \ldots \ldots \ldots \ldots \ldots \ldots$ & + & - & 一 & - & - \\
\hline $1 / 640 \quad \ldots \ldots \ldots \ldots \ldots \ldots \ldots$ & 一 & 一 & 一 & 一 & 一 \\
\hline \multicolumn{6}{|l|}{ Sérum négatif: } \\
\hline 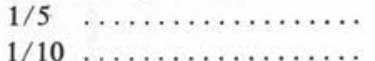 & - & - & - & - & 一 \\
\hline & - & & & & \\
\hline \multicolumn{6}{|l|}{ Sérum anti-flore : } \\
\hline $1 / 5 \quad \ldots \ldots \ldots \ldots \ldots \ldots \ldots$ & + & + & + & + & + \\
\hline $1 / 10 \quad \ldots \ldots \ldots \ldots \ldots \ldots$ & + & + & + & + & $\mp$ \\
\hline $1 / 20 \ldots \ldots \ldots \ldots \ldots \ldots$ & $\mp$ & + & + & $\mp$ & - \\
\hline $1 / 40 \ldots \ldots \ldots \ldots \ldots \ldots$ & $\mp$ & + & + & $\mp$ & 一 \\
\hline $1 / 80 \ldots \ldots \ldots \ldots \ldots \ldots$ & 一 & + & $\mp$ & - & - \\
\hline $1 / 160 \quad \ldots \ldots \ldots \ldots \ldots \ldots$ & - & $\mp$ & $\mp$ & - & 一 \\
\hline $1 / 320 \quad \ldots \ldots \ldots \ldots \ldots \ldots$ & 一 & - & - & 一 & - \\
\hline $1 / 640 \quad \ldots \ldots \ldots \ldots \ldots \ldots$ & 一 & 一 & 一 & 一 & 一 \\
\hline \multicolumn{6}{|l|}{ Sérum anti-amibes: } \\
\hline $1 / 5 \quad \ldots \ldots \ldots \ldots \ldots \ldots \ldots$ & + & - & - & + & + \\
\hline $1 / 10 \quad \ldots \ldots \ldots \ldots \ldots \ldots$ & + & - & - & + & + \\
\hline $1 / 20 \ldots \ldots \ldots \ldots \ldots \ldots$ & + & - & - & + & + \\
\hline $1 / 40 \ldots \ldots \ldots \ldots \ldots \ldots$ & + & - & 一 & $\mp$ & + \\
\hline $1 / 80 \quad \ldots \ldots \ldots \ldots \ldots \ldots$ & + & - & - & 0 & $\mp$ \\
\hline $1 / 160 \quad \ldots \ldots \ldots \ldots \ldots \ldots$ & $\mp$ & - & - & 0 & - \\
\hline
\end{tabular}

La R.F.C. révèle 3 fractions spécifiques de l'amibe $=$ F I, F IV, F V.

F II et F III correspondent à la flore associée.

\section{III) Hémagglutination.}

Les cinq fractions essayées dans la réaction d'hémagglutination confirment les résultats obtenus en R.F.C. 


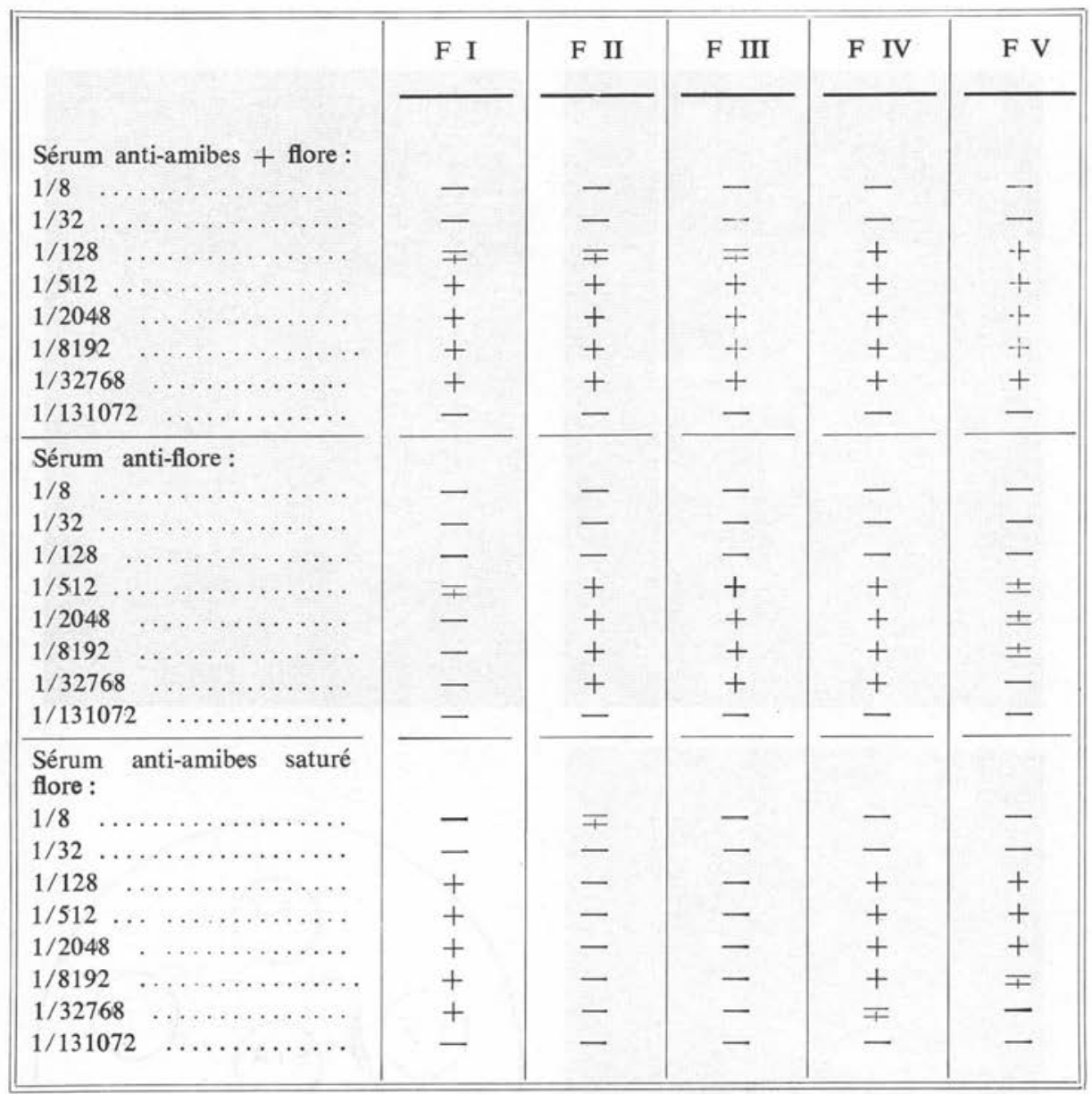

IV) Technique de double diffusion en gélose.

Les résultats obtenus sont reproduits dans les photos et graphiques de la planche II.

1) Technique d'Ouchterlony : une ligne de précipitation nette est obtenue avec la fraction F I et deux avec la fraction F V (fig. 4 de la planche II).

2) Technique de Oudin : trois fractions - F I, IV et V - donnent naissance à des lignes de précipitation, selon les schémas des fig. 1, 2 et 3 de la planche II. 

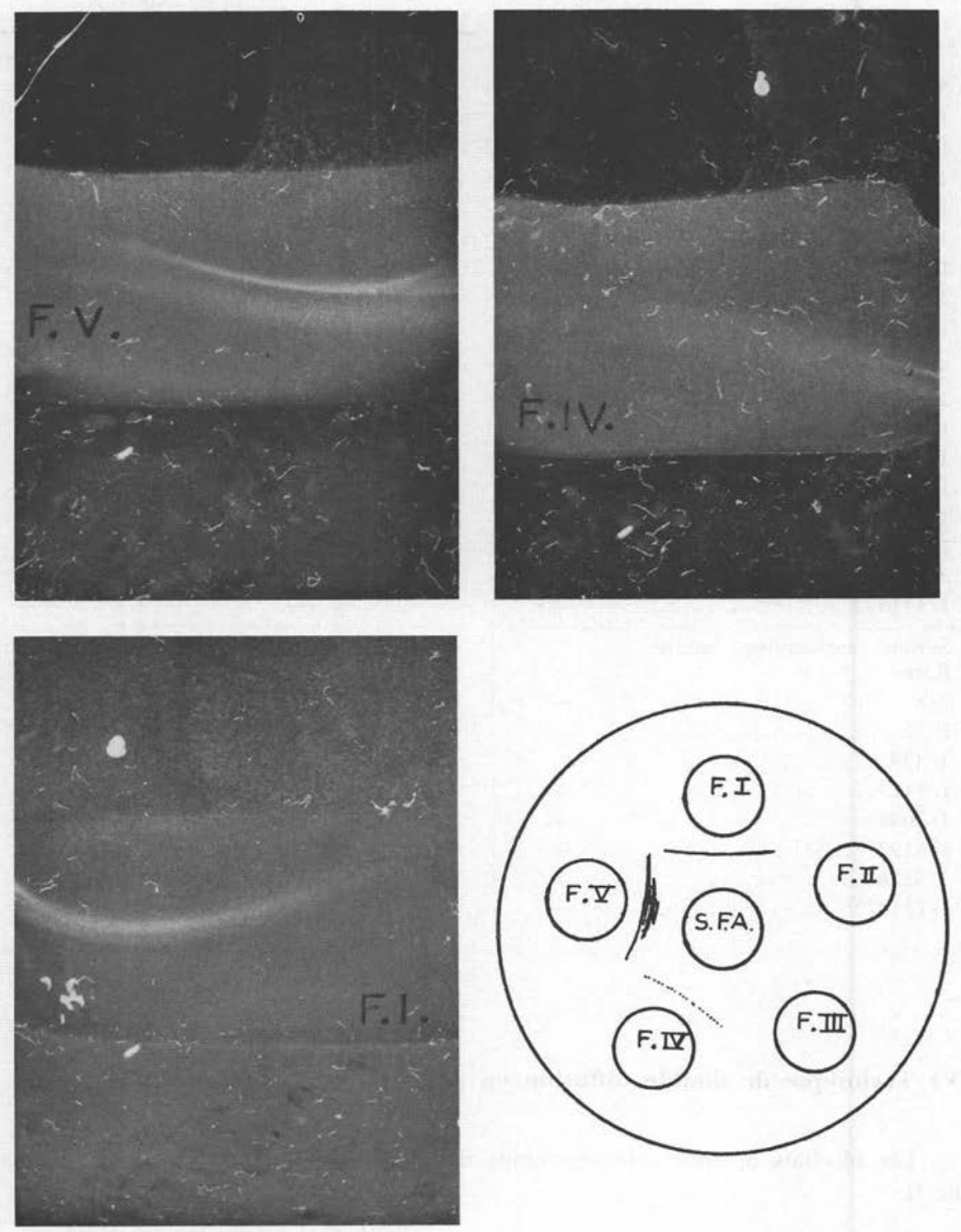

Planche II. - Immunodiffusion en cuves de Oudin et en boîte d'Ouchterlony des fractions séparées 


\section{Discussion}

Tous les résultats obtenus dans les différentes techniques conduisent aux mêmes résultats. Il apparaît que deux fractions sont immunologiquement spécifiques d' $E$. histolytica $=$ la F I et la F V.

La fraction IV fait preuve dans certaines techniques d'une faible réactivité. A l'examen de la courbe de fractionnement, il semble que cette activité serait liée à une mauvaise séparation des deux dernières fractions, une partie des composants actifs de la F V restant accrochés à la F IV. Ce phénomène s'étant reproduit à chaque fractionnement sur du Sephadex G. 200, un fractionnement ultérieur sur un gel de dimensions différentes devrait permettre de vérifier s'il s'agit réellement d'une seule fraction ou de deux avec des composants chimiques différents.

\section{Comparaison avec les résultats du fractionnement d'un antigène d'Entamoeba histolytica maintenues en eulture pure.}

Nous poursuivrons notre étude comparative avec les résultats obtenus par Thompson et coll. en 1968 (8).

Ces auteurs, utilisant un gel de dextrane identique à celui employé dans notre propre expérimentation (Sephadex G. 200), obtiennent à $280 \mathrm{~m} \mu$ une courbe présentant également cinq pics correspondant à cinq fractions dont deux majeures: F I et F V. Du point de vue activité immunologique, trois sont sélectionnées:

F I avec une activité ++++ ,

F II +

F III +

Pour pouvoir comparer cette courbe avec celle obtenue par nous-mêmes, il faut prendre des paramètres identiques.

Or, si le gel et l'éluant sont les mêmes, nous voyons que :

- pour Thompson, 100 tubes de la séparation, 3.000 gouttes, $186 \mathrm{ml}$;

- pour nous-mêmes, 100 tubes de la séparation, 5.000 gouttes.

Les hauteurs des colonnes étant différentes - $85 \mathrm{~cm}$ contre 45 - la vitesse de passage n'est pas non plus comparable. Les seuls points de comparaison ne peuvent être que les rapports de proportions entre les différentes fractions.

Après analyse des pics et évaluation des surfaces, il ressort que les cinq fractions obtenues sur notre colonne se replacent dans les 150 premiers tubes de l'élution de Thompson, notre fractionnement ayant été arrêté avant l'obtention des pics 4 et 5 de Thompson, dépourvus du reste d'activité immunologique. Nous recueillons donc ainsi deux pics de plus que les auteurs américains dans la même zone de P.M.

Nos résultats immunologiques ont fait apparaître que deux de ces fractions la III et la IV - correspondaient en fait à l'antigénicité de la flore. Il reste donc trois fractions - la 1, la 4 et la 5-à comparer aux fractions I, II et III de Thompson. Ces six fractions paraissent se comporter d'une manière superposable dans les trois 
tests sérologiques, utilisés de part et d'autre avec le même décalage d'une dilution pour la R.F.C. que celui constaté avec l'antigène brut total; la réaction d'hémagglutination, en revanche, présente une sensibilité un peu plus grande avec notre propre antigène.

Le tableau suivant résume les résultats comparatifs avec les fractions obtenues à partir des deux antigènes :

\begin{tabular}{|c|c|c|c|c|c|c|}
\hline \multirow[b]{3}{*}{ D.D.G. . } & \multicolumn{3}{|c|}{ Ag. culture mixte } & \multicolumn{3}{|c|}{ Ag. culture pure } \\
\hline & F I & F IV & F V & F I & F II & F III \\
\hline & 3 traits & 1 trait & 3 traits & 3 traits & 1 trait & 2 traits \\
\hline H.A. $\ldots$ & $1 / 32768$ & $1 / 8192$ & $1 / 2048$ & $\begin{array}{c}1 / 16384 \\
\text { fausse }+ \text { aux } \\
\text { faibles dilutions }\end{array}$ & $1 / 4096$ & $1 / 252$ \\
\hline R.F.C. . & $1 / 32$ & $1 / 4$ & 0 & $1 / 64$ & $\mp$ & $\mp$ \\
\hline
\end{tabular}

\section{CONCLUSION}

Après examen de ces résultats, nous pouvons donc conclure que le fractionnement sur une colonne de Sephadex G. 200 d'un antigène préparé à partir d'E. histolytica maintenues en culture mixte avec des bactéries, permet la séparation de trois fractions spécifiques, immunologiquement actives, comparables aux fractions séparées sur un même gel à partir de souches maintenues en culture pure.

Deux fractions correspondant à la flore associée sont en outre obtenues.

Les fractions sont spécifiques mais, dans l'un et l'autre cas, leur sensibilité est moindre que celle des antigènes bruts.

La présence des fractions F II et F III dues à la flore dans l'antigène total n'amène pas de fausses réactions positives à condition de pratiquer la saturation des sérums. Des confrontations ultérieures avec des sérums issus d'autres Protozoaires sont nécessaires afin de préciser la technique la plus rentable.

\section{Bibliographie}

(1) BÉNEX (J.). - Extraits antigéniques spécifiques obtenues à partir d'Entamoeba histolytica maintenues en culture mixte. I. Obtention, caractérisation chimique et immunologique des extraits. Ann. I.P. (sous presse).

(2) Thompson (P. E.), Graedel (S. K.), Schneider (C. R.), Stucki (W. P.), and Gordon (R. M.), 1968. - Préparation of standardized amoeba antigen from axenic cultures of Entamoeba histolytica. Bull. O.M.S., 39, 349-65. 